\title{
NEUTRALIZED TRANSPORT OF HIGH INTENSITY BEAMS
}

\author{
E. Henestroza ${ }^{\#}$, S. S. Yu, S. Eylon, P.K. Roy, A. Anders, W. Sharp, LBNL \\ P. Efthimion, E. Gilson, PPPL \\ D. Welch, D. Rose, MRC
}

\section{Abstract}

The NTX experiment at the Heavy Ion Fusion Virtual National Laboratory is exploring the performance of neutralized final focus systems for high perveance heavy ion beams. A converging ion beam at the exit of the final focus magnetic system is injected into a neutralized drift section. The neutralization is provided by a metal arc source and an RF plasma source. Effects of a "plasma plug", where electrons are extracted from a localized plasma in the upstream end of the drift section, and are then dragged along by the ion potential, as well as the "volumetric plasma", where neutralization is provided by the plasma laid down along the ion path, are both studied and their relative effects on the beam spot size are compared. Comparisons with 3-D PIC code predictions will also be presented.

\section{INTRODUCTION}

A driver beam is transported in the final focus section through several magnetic quadrupoles, and is then allowed to drift ballistically through neutralizing plasma in a low-density (millitorr) gas onto the target. There are nonlinear processes both in the magnetic section as well as in the neutralized transport section. To investigate these phenomena, the Neutralized Transport Experiment (NTX) has begun at LBNL [1]. The experimental setup (Fig. 1) consists of three major sections, a low-emittance potassium gun [2], a magnetic transport section with 4 pulsed quadrupoles [3,4], and a one-meter long drift section with plasma neutralization.

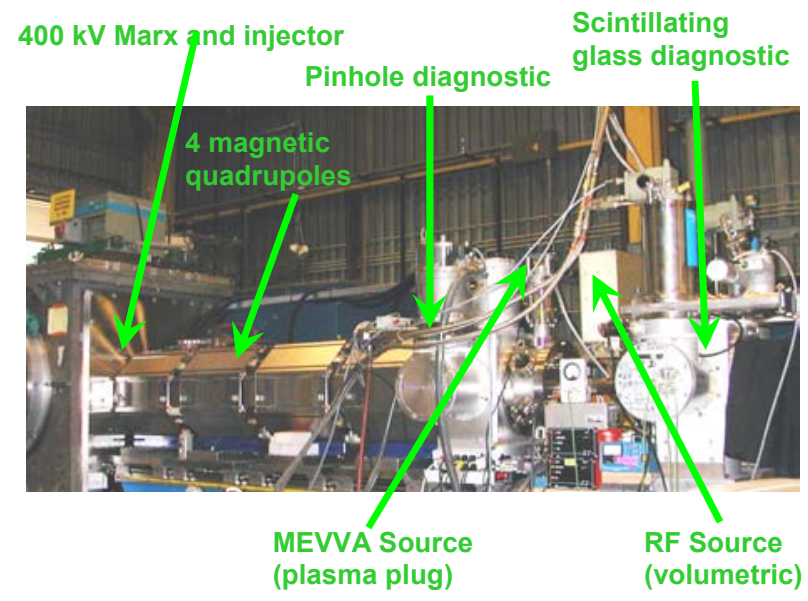

Figure 1: Neutralized Transport Experiment (NTX).

\footnotetext{
"EHENESTROZA@lbl.gov
}

*This work has been performed under the auspices of the US DOE by UC-LBNL under contract DE-AC03-76SF00098, for the Heavy Ion Fusion Virtual National Laboratory.
The MRC electromagnetic code LSP has been used to specify the requirements for the plasma source, as well as the predicted degree of neutralization as a function of the various beam and plasma parameters. The PIC simulation code WARP has also been used to simulate the beam dynamics for specific degrees of neutralization.

\section{PLASMA NEUTRALIZATION}

Neutralization is essential for focusing heavy ion beams onto target. Figure 2 shows a conceptual layout of a neutralization system. For NTX the plasma plug is a MEVVA plasma source, the volumetric plasma is supplied by an RF source and the target is a scintillator imaged by a CCD camera.

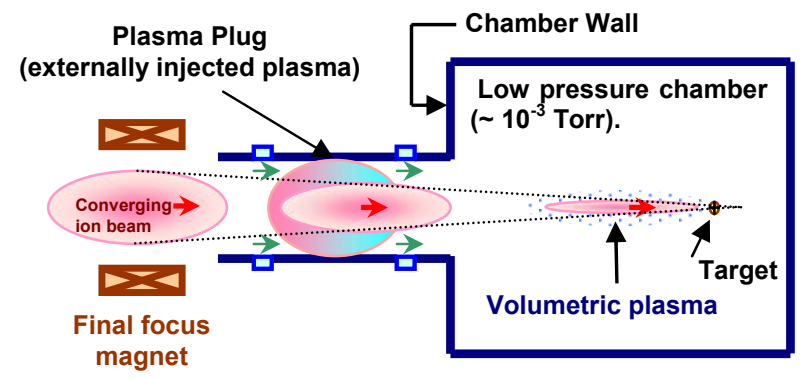

Figure 2: Neutralization system layout.

The MEVVA (Fig. 3) and the Argon plasma (Fig. 4) sources deliver reproducible plasma densities ten times greater than the beam density, sufficient for a range of plasma-plug and volumetric neutralization experiments.

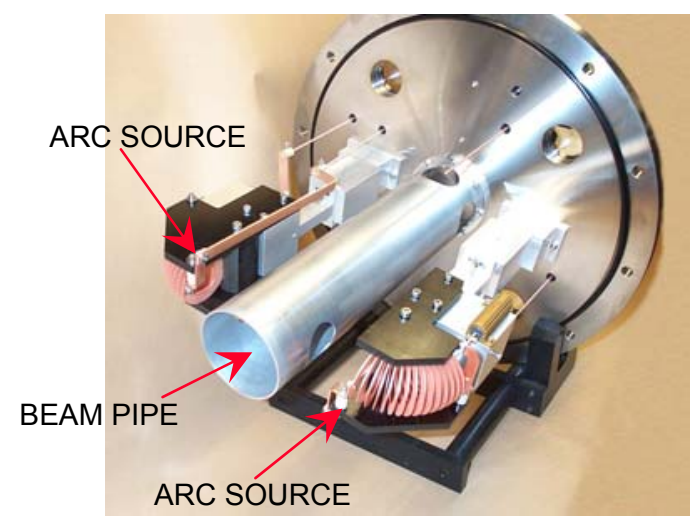

Figure 3: MEVVA plasma source. 


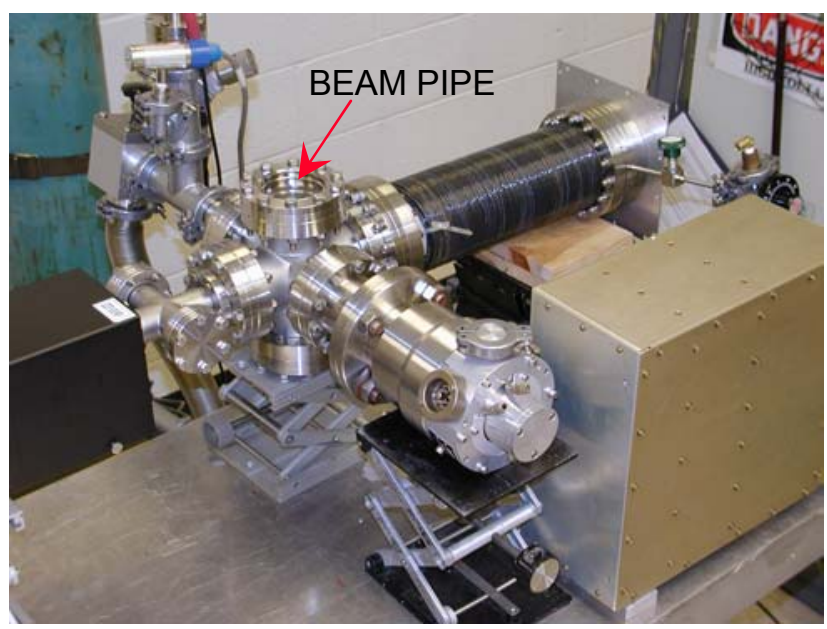

Figure 4: Argon RF plasma source.

\section{BEAM DYNAMICS}

The NTX injector can deliver high brightness beams in a range of $220-340 \mathrm{keV}, 15-30 \mathrm{~mA}$, with a normalized emittance of $\sim 0.05 \pi-\mathrm{mm}-\mathrm{mr}$ and a uniform density profile. The quadrupole fields in the final focus system are chosen to obtain a beam of one-meter focal length (20 $\mathrm{mm}$ radius and $20 \mathrm{mr}$ convergence) at the entrance to the neutralization region for the nominal energy $(300 \mathrm{keV}, 25$ $\mathrm{mA}$ ). Optical scintillators imaged by CCD cameras and mechanical slit scanners [5] were used to measure the beam profiles and phase space distributions of the beam at the end of the final focus lattice. Figure 5 shows agreement between the measured and calculated beam profiles for the nominal energy and quadrupole field configuration.

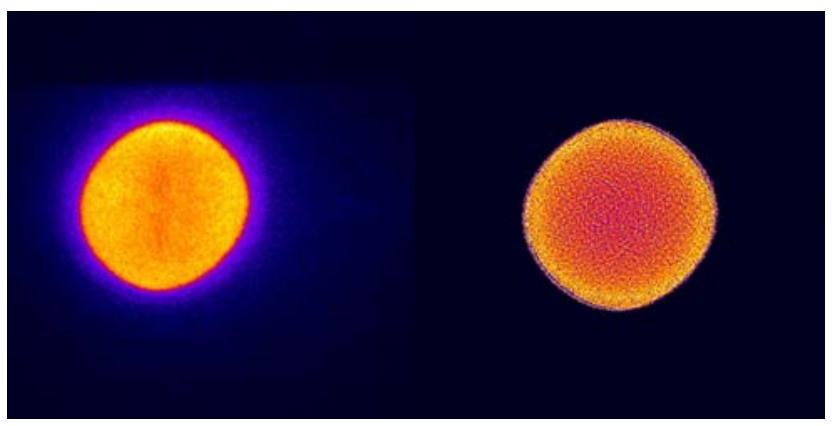

Figure 5: CCD camera image and WARP simulation results of converging beam profiles at entrance to the neutralization region.

The slight distortion of the measured beam profile is due to a small rotation $(5 \mathrm{mr})$ of one of the quadrupoles. The beam is uniform with a narrow rim due to field aberrations and the final normalized emittance of the beam is $0.1 \pi-\mathrm{mm}-\mathrm{mr}$.

\section{Non-neutralized drift}

As a preliminary test of the NTX system, the beam was injected into a vacuum pipe without any neutralization. Figure 6 shows the measured beam profiles at 1 meter downstream from the exit of the final focus system. The energy varies from 260 to $300 \mathrm{keV}$, and the beam size is in good agreement with WARP simulations.

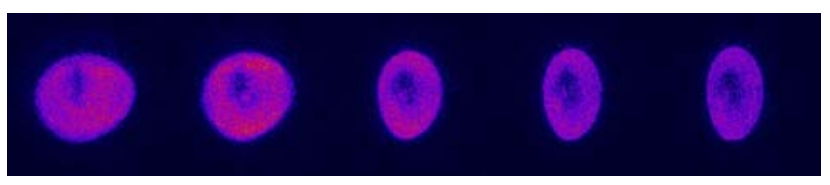

Figure 6: Beam profiles 1 meter downstream from the exit of the final focus system for non-neutralized drift.

\section{Neutralized transport}

The low emittance ( $\sim 30 \pi$-mm-mr unnormalized) of the beam at the entrance to the neutralized region allows for the beam to be focused to a small spot size $(1-2 \mathrm{~mm}$ radius) at the focal point for neutralized ballistic transport. Figure 7 shows the measured dependence of beam spot size at the focal plane on neutralization mechanism.

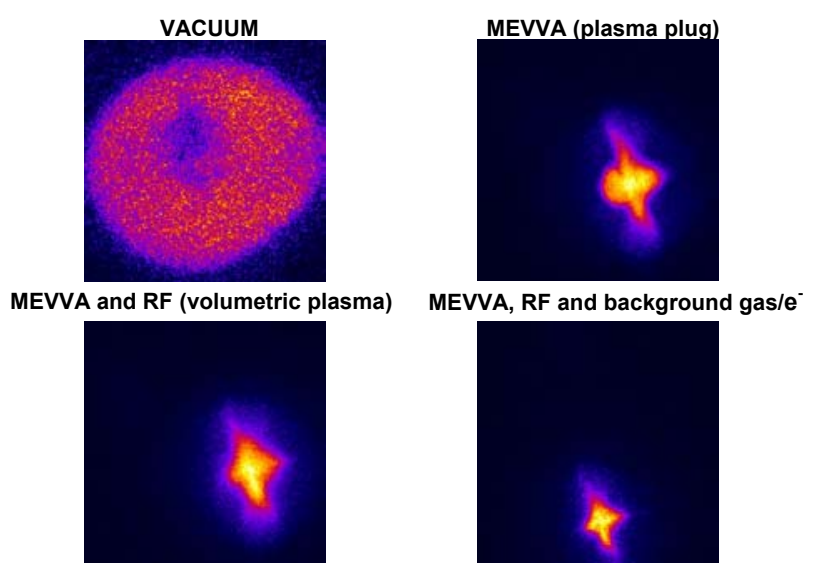

Figure 7: Spot size dependence on neutralization mechanism. Image box size is $4 \mathrm{~cm} \times 4 \mathrm{~cm}$ squares.

LSP simulations of NTX including plasma plug and volumetric neutralization were performed starting from beam initial conditions at the entrance to the neutralization region. In one series (Fig. 8) of LSP simulations, the initial condition is the final phase space distribution as calculated by WARP for the transport of the NTX beam from the gun to the exit of the final focus system. There is qualitative agreement with measured spot sizes. The measurement of the detailed structure of the phase space distribution in 4-dimensional phase space seems essential in order to run more realistic simulations of the beam focusing to a small spot when drifting through neutralizing plasma [4]. 


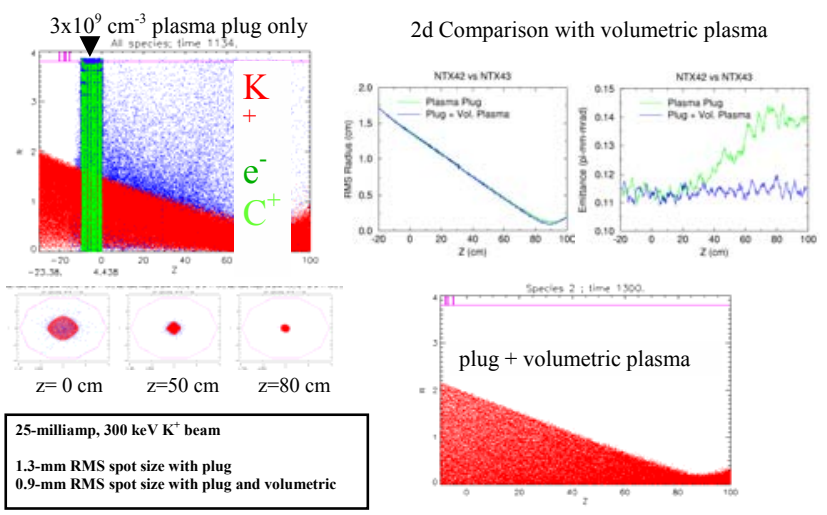

Figure 8: WARP-LSP NTX simulation with plasma plug neutralization.

\section{ANALOG SIMULATION OF FULLY NEUTRALIZED BEAM TRANSPORT}

We are developing a new technique [4] to measure the 4-dimensional phase space distribution of the beam using the beam imaging diagnostics. The phase space distribution $\mathrm{f}\left(\mathrm{x}, \mathrm{y}, \mathrm{x}^{\prime}, \mathrm{y}^{\prime}\right)$ can be measured by scanning the beam with a small pinhole (20 mils in diameter) and letting the transmitted beamlet to travel a long distance ( $\sim 1$ meter) before striking the scintillator where an image is taken. The position of the pinhole defines the coordinates $\mathrm{x}$ and $\mathrm{y}$, and from the image we can extract the density distribution of $x^{\prime}$ and $y^{\prime}$. This technique will be used to measure the detailed structure of the phase space distribution.

The same diagnostics system can be used to simulate the effect of full neutralization on a beam, since each beamlet going through a pinhole only carries the information about the phase space distribution at a given location without being perturbed by the space charge of the full beam. The superposition of all the pinhole images at a given location can be compared with a beam transport calculation where the space charge is turned off at the pinhole-scan plane. Figure 9 shows a comparison between this "analog simulation" and WARP; the agreement is excellent and shows that the emittance is low enough to get a small spot ( $\sim 1 \mathrm{~mm}$ radius).

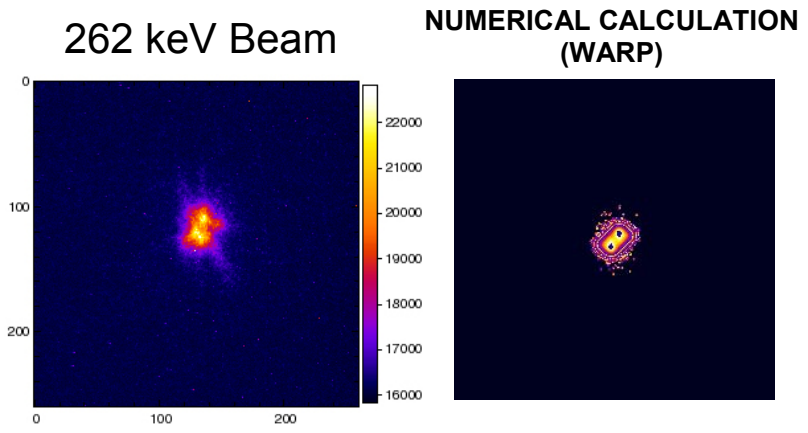

Figure 9: Analog (pinhole) simulation and WARP calculation of fully neutralized beam focused to a small spot. Image box size is $4 \mathrm{~cm} \mathrm{b \times 4} \mathrm{cm}$ squares.

\section{CONCLUSIONS}

Initial experiments of beam transport through the NTX neutralization section have produced a small spot at the focal plane consistent with numerical and analog calculations. Further measurements to obtain the 4-dimensional phase space distribution at the exit of the final focus system will provide the initial condition for more realistic simulation of the beam dynamics when ballistically focused through neutralizing plasma.

\section{REFERENCES}

[1] S. S. Yu et al., "Focusing and Neutralization of Intense Ion Beams", Proc. PAC '03, TOAA001.

[2] S. Eylon et al., "High brightness potassium source for the HIF neutralized transport experiment", Proc. PAC '03, WPPG012.

[3] D. Shuman et al., "Magnetic lattice for the HIF neutralized transport experiment", Proc. PAC '03, WPPG017.

[4] E. Henestroza et al., "Final Focus System for High Intensity Beams", Proc. PAC '03, WPPG013.

[5] F.M. Bieniosek et al., "Beam imaging diagnostics for heavy ion beam fusion experiments", Proc. PAC '03, WPPB050. 